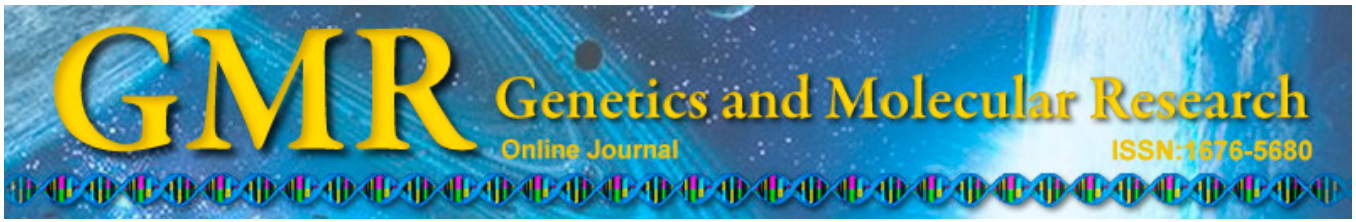

\title{
MicroRNAs as novel biomarkers for the differentiation of malignant versus benign thyroid lesions: a meta-analysis
}

\author{
G.J. Zhou, M. Xiao, L.N. Zhao, J.G. Tang and L. Zhang \\ Department of Otolaryngology-Head and Neck Surgery, Hangzhou, China \\ Corresponding author: L. Zhang \\ E-mail: leizhang_srrs@yeah.net
}

Genet. Mol. Res. 14 (3): 7279-7289 (2015)

Received June 9, 2014

Accepted April 6, 2015

Published July 3, 2015

DOI http://dx.doi.org/10.4238/2015.July.3.3

\begin{abstract}
The aim of this meta-analysis was to systematically evaluate the diagnostic accuracy of microRNAs (miRNAs) in distinguishing malignant thyroid lesions from benign ones and to determine the potential of miRNAs as diagnostic biomarkers for thyroid cancer. The random-effect model was used to summarize the pooled estimates of diagnostic accuracy, including sensitivity, specificity, positive likelihood ratio (PLR), negative likelihood ratio (NLR), and diagnostic odds ratio (DOR). The summary receiver-operating characteristic curve (SROC) and area under the SROC curve (AUC) were used to further evaluate the overall diagnostic value. Overall, 20 studies from 7 articles, including 266 thyroid cancer patients and 277 controls with benign thyroid disease, were available for analysis. The pooled sensitivity, specificity, PLR, NLR, and DOR were: $0.78(95 \% \mathrm{CI}$ $=0.74-0.81), 0.73(95 \% \mathrm{CI}=0.69-0.77), 3.17(95 \% \mathrm{CI}=2.28-4.40)$, $0.30(95 \% \mathrm{CI}=0.23-0.39)$, and $12.6(95 \% \mathrm{CI}=8.26-19.4)$, respectively, and the AUC value was 0.85 . The multiple miRNA assay yielded better diagnostic performance than the single miRNA assay, with sensitivity of 0.90 versus 0.75 , specificity of 0.86 versus 0.71 , PLR of 6.14 versus 2.71 , NLR of 0.13 versus 0.36 , DOR of 44.5 versus 8.81 , and AUC of
\end{abstract}


0.95 versus 0.82 , suggesting that the multiple miRNA assay is a more credible method for thyroid cancer detection. In summary, miRNA assays, especially multiple miRNA assays, may play an important role as a second-line diagnostic tool to improve the diagnostic accuracy of fine needle aspiration biopsy in indeterminate lesions. However, further studies are warranted to confirm our findings.

Key words: MicroRNAs; Thyroid cancer; Meta-analysis; Diagnosis

\section{INTRODUCTION}

Thyroid cancer, which typically presents in the form of a thyroid nodule, is the most common endocrine malignancy. In the past decade, the incidence of thyroid cancer has increased by 2.3 -fold with annual rates of 5.4\% in men and 6.5\% in women (Siegel et al., 2014). Most thyroid cancers are derived from follicular cells, and consist of conventional papillary thyroid carcinoma (PTC), follicular variant of PTC (FPTC), follicular thyroid carcinoma (FTC), Hürthle cell thyroid carcinomas, and anaplastic thyroid carcinoma (ATC) (Shibru et al., 2008; Vriens et al., 2009; Pallante et al., 2010). The diagnostic rate of thyroid nodules is over 7\% in the adult population; however, most thyroid nodules are benign, and only $5 \%$ of thyroid nodules are proven to be malignant (Dean and Gharib, 2008). For benign lesions, lifelong thyroid hormone replacement therapy can take the place of total thyroidectomy to reduce physical injury and to avoid surgical morbidity of patients (Mazeh et al., 2013). Therefore, although the 5- and 10-year survival rates of total thyroidectomy are excellent, diagnostic accuracy for distinguishing benign nodules from malignant ones may help with reducing unnecessary thyroid resections and improving clinical outcomes.

Currently, fine needle aspiration biopsy (FNAB) is the recommended approach for evaluating patients with thyroid nodules (Gharib et al., 2006). Based on different malignancy risks diagnosed by FNAB, 6 classification categories were identified: benign ( $0-3 \%$ malignancy risk), follicular lesion of undetermined significance (5-15\%), follicular neoplasm or suspicious for a follicular neoplasm (15-30\%), suspicious for malignancy (60-75\%), malignant $(97-99 \%)$, and undetermined (Layfield et al., 2010). With a relatively high diagnostic accuracy (65-98\% sensitivity and $72-100 \%$ specificity) (Gharib et al., 2006), approximately $70-80 \%$ of patients can be definitively categorized as having benign or malignant nodules by FNAB results, whereas the remaining $20-30 \%$ of lesions are interpreted to be indeterminate as the cytological features are inconclusive (Kato and Fahey, 2009; Lewis et al., 2009; Wang et al., 2011). When lesion results are interpreted as indeterminate, thyroidectomy is commonly recommended as a second surgical procedure, because indeterminate may be consistent with malignancy (Faquin and Baloch, 2010; Layfield et al., 2010). However, only 20-30\% of the indeterminate lesions have a malignancy risk because up to $80 \%$ of indeterminate lesions are benign, making a significant number of surgeries potentially avoidable (Khan et al., 2013; Lee et al., 2013). A combination of other diagnostic procedures have been used to solve the problem, such as the safe, sensitive, and straightforward ultrasonography method; however, this method is only effective with suspicious thyroid nodules, and is ineffective with improving the accuracy of FNAB cytology in indeterminate lesions (Banks et al., 2008; Dean and Gharib, 2008). Furthermore, no efficient immunohistochemical and molecular markers can reliably 
determine which of these patients can avoid unnecessary thyroidectomy, although great efforts have been made in recent years toward this end (Shibru et al., 2008; Stang and Carty, 2009; Vriens et al., 2009; Chudova et al., 2010; Kouniavsky and Zeiger, 2010; Adeniran et al., 2011). Thus, safe and accurate novel biomarkers are still urgently needed as a second-line diagnostic tool to determine malignancy with a high accuracy level for patients with indeterminate lesions.

MicroRNAs (miRNAs), which are a family of recently discovered noncoding RNAs of 18-24 nucleotides in length, function primarily to modulate the translation of various protein-coding genes (Engels and Hutvagner, 2006; Visone et al., 2007). A single proteincoding gene can be potentially targeted by multiple different miRNAs, and a single miRNA can also potentially target hundreds of protein-coding genes (Wiemer, 2007; Reddy et al., 2010). Recent studies have demonstrated that dysregulation of miRNAs is commonly found in malignancies, and there is substantial evidence that miRNAs may be involved in tumorigenesis (Martinez and Dimaio, 2011; Subramanyam and Blelloch, 2011; Wilmott et al., 2011). Furthermore, miRNA signatures have been investigated in thyroid cancer, and researchers have found that miRNA expression profiles were able to differentiate benign from malignant thyroid lesions, suggesting that miRNAs might serve as diagnostic biomarkers for thyroid cancer (Keutgen et al., 2012; Kitano et al., 2011, 2012; Shen et al., 2012; Vriens et al., 2012; Mazeh et al., 2011, 2013). Through investigating the expression of 34 miRNAs in benign and malignant thyroid neoplasms, Kitano et al. (2011) demonstrated that miR-7 and miR-126 could significantly differentiate malignant thyroid tumors from benign ones with high diagnostic accuracy [area under the receiver operating characteristic curve $(A U C)=0.81$ and 0.77, respectively]. Similarly, Keutgen et al. (2012) showed that a panel of 4 miRNAs (miR-328, miR-222, miR-21, and miR-197) could distinguish malignant from benign indeterminate FNAB thyroid lesions with sensitivity of $100 \%$ and specificity of $86 \%$.

Although a number of studies have explored the expression of miRNAs in FNAB lesions and have determined the potential use of miRNAs for differentiating malignant from benign indeterminate FNAB lesions, varied accuracy rates were obtained without consensus (Keutgen et al., 2012; Kitano et al., 2011, 2012; Shen et al., 2012; Vriens et al., 2012; Mazeh et al., 2011, 2013). Therefore, we conducted this meta-analysis to assess the diagnostic accuracy of miRNAs for differentiating benign from malignant nodules on FNAB samples, and to determine the potential value of miRNAs to serve as a second-line diagnostic tool in thyroid cancer detection.

\section{MATERIAL AND METHODS}

\section{Search strategy}

Search terms including ("thyroid cancer" or "thyroid carcinoma") and ("microRNAs" or "miRNA" or "miRs") and ("diagnosis" or "ROC curve" or "sensitivity" or "specificity") were used in the systematic literature research. Eligible studies were searched in the PubMed, Cochrane Library, EMBASE, and Chinese National Knowledge Infrastructure databases up to March 26, 2014 without language restriction. We also manually searched other resources for any relevant records potentially missed. 


\section{Selection criteria}

Eligible studies were required to meet the following criteria: 1) studies on miRNA expression in differentiating malignant from benign thyroid lesions; 2) provides sensitivity and specificity or can calculate them from the literature; 3) used FNAB results as the gold standard for the diagnosis of thyroid lesions; 4) not reviews, editorials, or case reports; 5) the control groups were diagnosed with benign thyroid disease.

\section{Data extraction and quality evaluation}

Two reviewers independently extracted data from studies included. Data retrieved from these articles included first author, year of publication, type of study, number of patients and controls, miRNA expression signature, specimen, test method, and sensitivity and specificity data. The quality of the studies included was assessed using the revised Quality Assessment of Diagnostic Studies-2 (QUADAS-2) metric (Whiting et al., 2003). The tool is based on 7 items concerning patient selection bias, index testing, and reference standards, with each item having the responses of 'yes', 'no', or 'unclear' to judge the risk of bias and applicability.

\section{Statistical analysis}

All statistical analyses were conducted using the Meta-Disc 1.4 software (Zamora et al., 2006). The random-effect model was applied to summarize the pooled estimates of diagnostic accuracy for the microRNA assay, including sensitivity, specificity, positive likelihood ratio (PLR), negative likelihood ratio (NLR), and diagnostic odds ratio (DOR). The chi-square test and $I^{2}$ statistic were used to quantify the degree of between-study heterogeneity (Higgins et al., 2003). Summary receiver-operating characteristic curve (SROC) and the AUC were used to further evaluate overall diagnostic value (Walter, 2002). The $Q^{*}$ index, which is the point closest to the ideal top-left corner of the SROC space, shows the best combination of sensitivity and specificity (Walter, 2002).

\section{RESULTS}

\section{Characteristics and quality of the studies included}

The literature screening process is shown in Figure 1. In total, 93 potentially relevant records were identified through database searching $(\mathrm{N}=89)$ and other sources $(\mathrm{N}=4)$. Seventy-five records remained after duplicates were removed. After which, 36 records were further removed, among which 22 were abstracts, reviews, or meta-analyses, with the other 14 not related to this research topic. Through further full-text screening of the remaining 39 records for eligibility, 32 records were excluded, as 12 of them were prognostic studies and 20 were without sufficient data. Ultimately, 7 records (Keutgen et al., 2012; Kitano et al., 2011, 2012; Shen et al., 2012; Vriens et al., 2012; Mazeh et al., 2011, 2013) were available for the meta-analysis.

The characteristics of the studies included, along with QUADAS-2 scores, are shown in Table 1. Overall, 20 studies from the 7 records (an article may be separated into several studies if it investigated more than one miRNAs), including 266 thyroid cancer patients and 
277 controls with benign thyroid disease, were available for analysis. The studies included were published from 2011 to 2013; of these, 5 were conducted in the USA (Keutgen et al., 2012; Kitano et al., 2011, 2012; Shen et al., 2012; Vriens et al., 2012), and 2 were conducted in Israel (Mazeh et al., 2011, 2013), and all the patients and controls were of Caucasian descent. All miRNAs involved in the meta-analysis were analyzed using the method of quantitative reverse transcription polymerase chain reaction (qRT-PCR), and the category of specimen was fine needle aspiration sample. Of the 20 studies, 16 focused on the diagnostic accuracy of a single microRNA, while the other 4 studies evaluated the diagnostic value of multiple microRNAs as biomarkers in thyroid cancer detection. All the eligible studies were of moderately high quality with QUADAS-2 scores above 4 points.

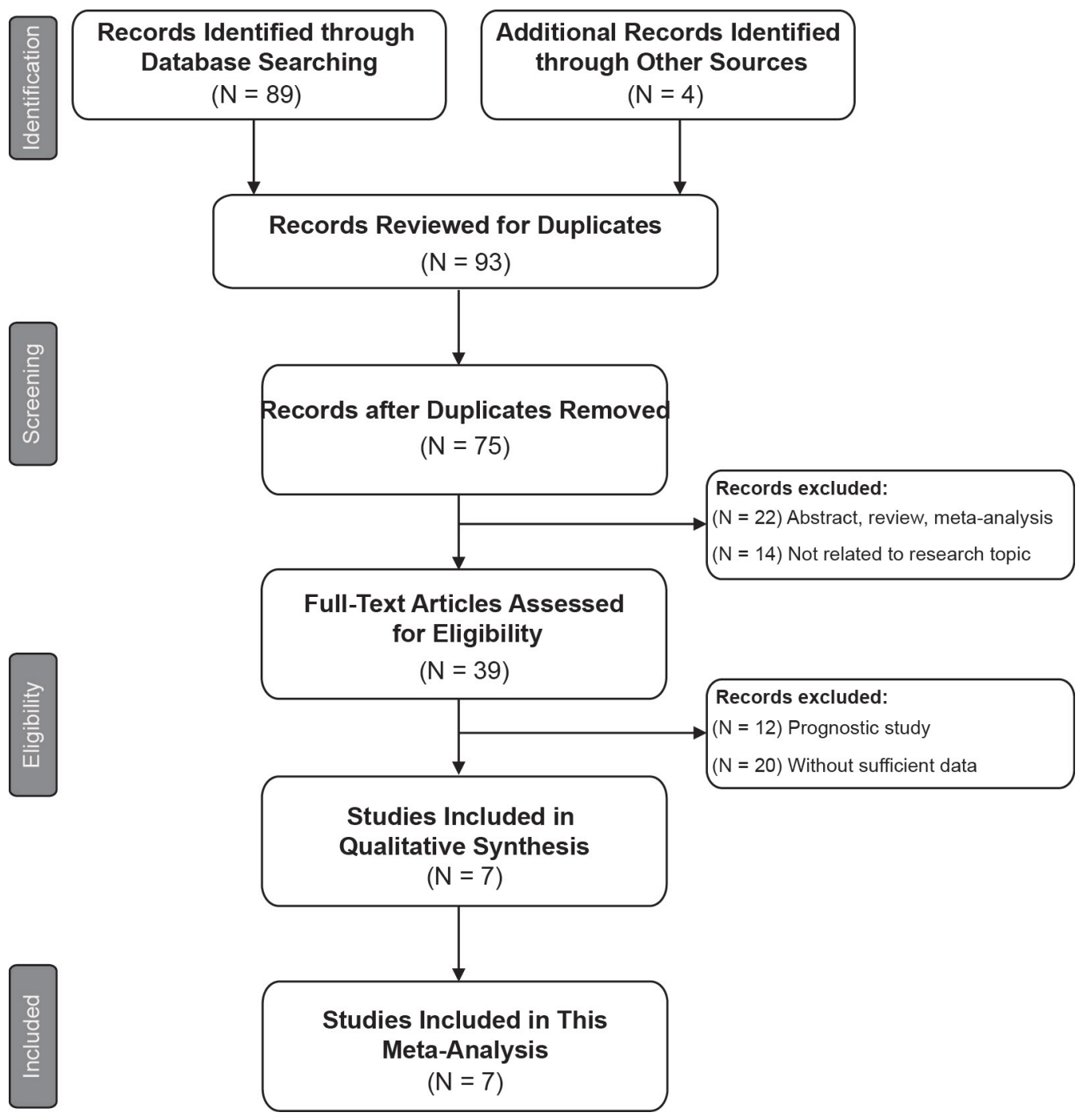

Figure 1. Literature screening process. 
G.J. Zhou et al.

7284

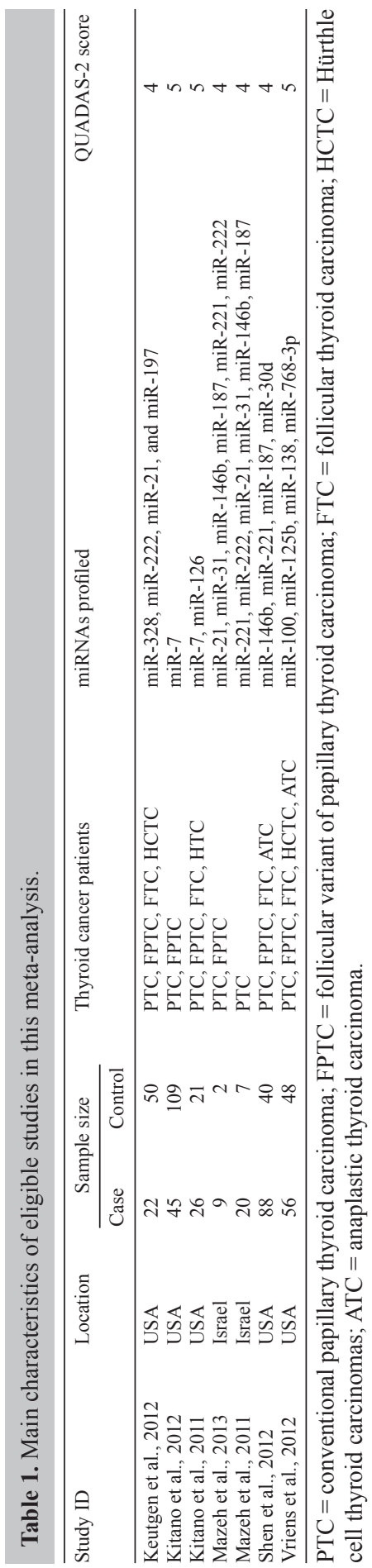

Genetics and Molecular Research 14 (3): 7279-7289 (2015) 


\section{Diagnostic accuracy}

Summary results of diagnostic performance for miRNAs assays in thyroid cancer detection are presented in Table 2 . The pooled sensitivity was $0.78(95 \% \mathrm{CI}=0.74-0.81)$ and specificity was $0.73(95 \% \mathrm{CI}=0.69-0.77)$, while the AUC value was 0.85 . The pooled PLR was $3.17(95 \% \mathrm{CI}=2.28-4.40)$ and the pooled NLR was $0.30(95 \% \mathrm{CI}=0.23-0.39)$. The pooled DOR was $12.6(95 \% \mathrm{CI}=8.26-19.4)$. These results indicated a relatively high accuracy of miRNA assays in differentiating thyroid cancer from benign thyroid disease. The betweenstudy heterogeneity was assessed by the $I^{2}$ index. The $I^{2}$ results were $71.2 \%$ for sensitivity (P $=0.001), 79.2 \%$ for specificity $(\mathrm{P}=0.001), 76.9 \%$ for PLR $(\mathrm{P}=0.001), 62.6 \%$ for NLR $(\mathrm{P}=$ $0.001)$, and $36.3 \%$ for DOR ( $\mathrm{P}=0.054)$, indicating significant heterogeneity between studies. Thus, the random-effect model was chosen for this meta-analysis.

\begin{tabular}{|c|c|c|c|}
\hline Analysis & Overall & Single miRNA & Multiple miRNAs \\
\hline No. of studies & 20 & 16 & 4 \\
\hline Sensitivity $(95 \% \mathrm{CI})$ & $0.78(0.74-0.81)$ & $0.75(0.71-0.79)$ & $0.90(0.83-0.95)$ \\
\hline$I^{2}\left(\mathrm{P}_{\mathrm{t}}\right.$-value $)$ & $71.2(0.001)$ & $70.3(0.001)$ & $0.0(0.815)$ \\
\hline Specificity $(95 \% \mathrm{CI})$ & $0.73(0.69-0.77)$ & $0.71(0.66-0.75)$ & $0.86(0.77-0.92)$ \\
\hline$I^{2}(\mathrm{P}$-value $)$ & $79.2(0.001)$ & $81.0(0.001)$ & $0.0(0.492)$ \\
\hline $\operatorname{PLR}(95 \% \mathrm{CI})$ & $3.17(2.28-4.40)$ & $2.71(1.98-3.73)$ & $6.14(3.58-10.5)$ \\
\hline$I^{2}\left(\mathrm{P}_{-}\right.$-value $)$ & $76.9(0.001)$ & $73.6(0.001)$ & $0.0(0.639)$ \\
\hline NLR $(95 \%$ CI $)$ & $0.30(0.23-0.39)$ & $0.36(0.28-0.45)$ & $0.13(0.07-0.23)$ \\
\hline$I^{2}(\mathrm{P}-$ value $)$ & $62.6(0.001)$ & $50.2(0.012)$ & $0.0(0.699)$ \\
\hline DOR $(95 \%$ CI $)$ & $12.6(8.26-19.4)$ & $8.81(6.19-12.5)$ & $44.5(19.0-104)$ \\
\hline$I^{2}(\mathrm{P}$-value $)$ & $36.3(0.054)$ & $8.3(0.359)$ & $0.0(0.556)$ \\
\hline AUC & 0.85 & 0.82 & 0.95 \\
\hline
\end{tabular}

$\mathrm{CI}=$ confidence interval, $\mathrm{PLR}=$ positive likelihood ratio, $\mathrm{NLR}=$ negative likelihood ratio, $\mathrm{DOR}=$ diagnostic odds ratio, $\mathrm{AUC}=$ area under the curve, $\mathrm{P}_{h}=\mathrm{P}$ value of heterogeneity.

\section{Subgroup analysis}

A subgroup analysis was also conducted between the single miRNA and multiple miRNA assays. For the single miRNA assay, the pooled sensitivity and specificity were 0.75 $(95 \% \mathrm{CI}=0.71-0.79)$ (Figure 2A) and $0.71(95 \% \mathrm{CI}=0.00-0.75)$ (Figure $2 \mathrm{~B})$. The pooled PLR, NLR, and DOR were $2.71(95 \% \mathrm{CI}=1.98-3.73), 0.36(95 \% \mathrm{CI}=0.28-0.45)$, and 8.81 $(95 \% \mathrm{CI}=6.19-12.5)$, respectively (Table 2$)$. The $I^{2}$ results were $70.3 \%$ for sensitivity $(\mathrm{P}=$ $0.001), 81.0 \%$ for specificity $(\mathrm{P}=0.001), 73.6 \%$ for PLR $(\mathrm{P}=0.001), 50.2 \%$ for NLR $(\mathrm{P}=$ $0.012)$, and $8.3 \%$ for DOR $(\mathrm{P}=0.359)$, indicating significant heterogeneity among studies. For the multiple miRNA assay, the pooled sensitivity and specificity were $0.90(95 \% \mathrm{CI}=0.83$ $0.95)$ (Figure $2 \mathrm{C}$ ) and $0.86(95 \% \mathrm{CI}=0.77-0.92)$, respectively (Figure 2D). The pooled PLR was $6.14(95 \% \mathrm{CI}=3.58-10.5)$, the pooled NLR was $0.13(95 \% \mathrm{CI}=0.07-0.23)$, and the pooled DOR was $44.5(95 \% \mathrm{CI}=19.0-104)$ (Table 2$)$. The AUC was 0.82 for a single miRNA (Figure $3 \mathrm{~A}$ ) and 0.95 for multiple miRNAs (Figure 3B), indicating that multiple miRNAs display a relatively good ability to distinguish thyroid cancer patients. Notably, compared with the single miRNA assay, the overall outcomes were better with the multiple miRNA assay. Thus, multiple miRNAs may be promising biomarkers in clinical practice. 
(A) Single miRNA

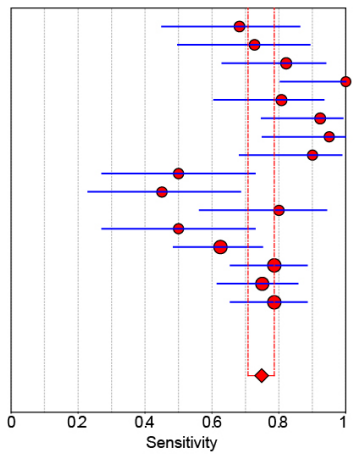

(C) Multiple miRNAs

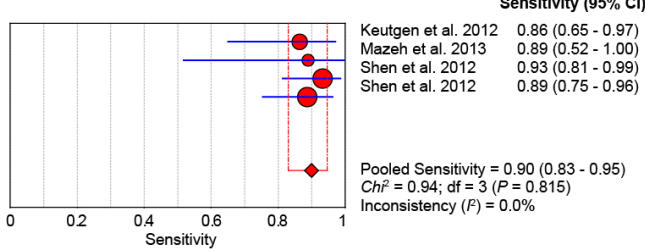

(B) Single miRNA

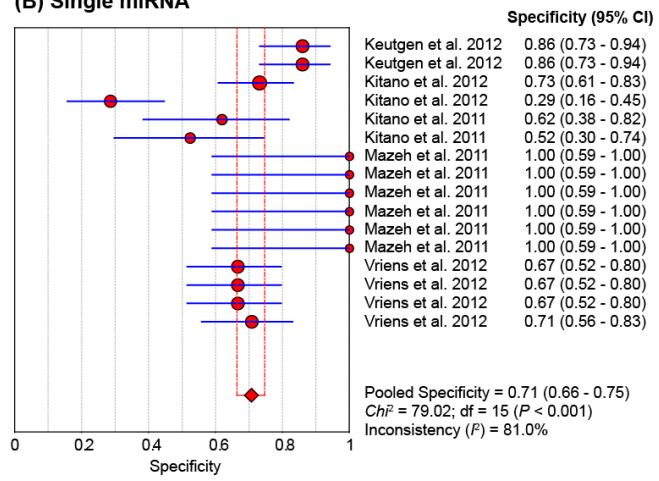

(D) Multiple miRNAs

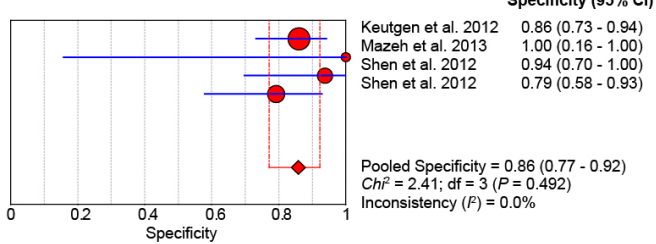

Figure 2. Forest plot showing study-specific (right-axis) and mean sensitivity and specificity with corresponding heterogeneity statistics. A. Sensitivity and B. specificity for single miRNAs. C. Sensitivity and D. specificity for multiple miRNAs.

(A) Single miRNA

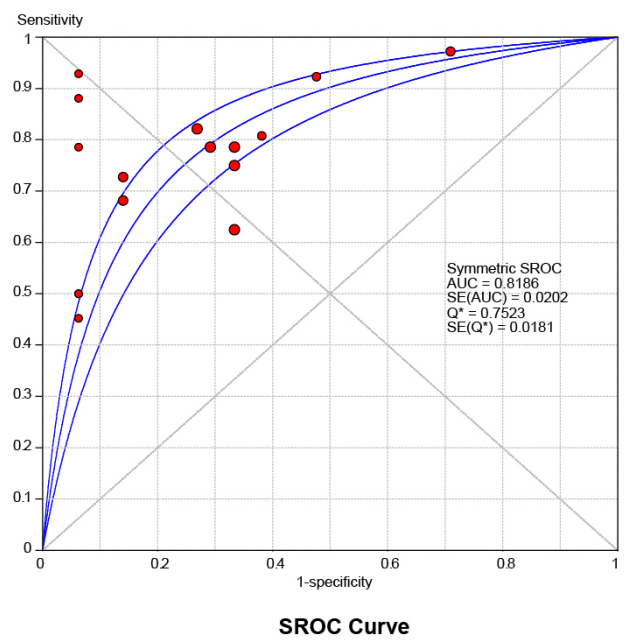

(B) Multiple miRNAs

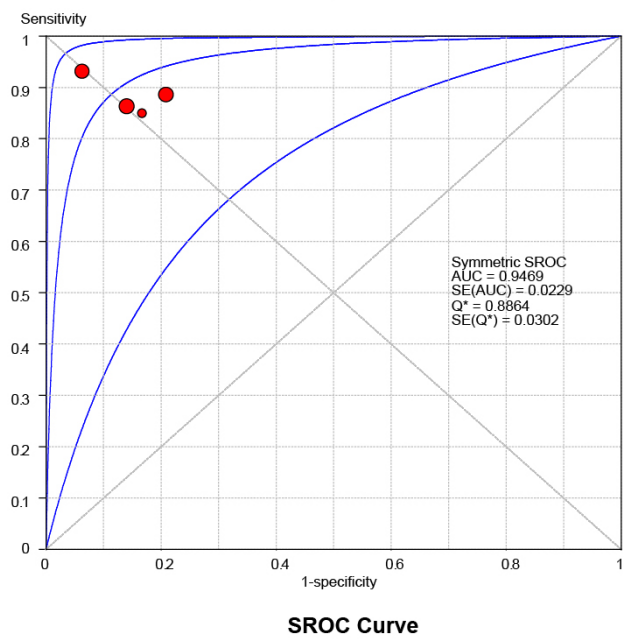

Figure 3. Symmetric receiver operating characteristic (SROC) curves for diagnosis by single (A) and multiple (B) miRNAs.

\section{DISCUSSION}

The prevalence of thyroid nodules in the population is increasing; nevertheless, only 
a small fraction of all thyroid nodules harbor malignant disease (Dean and Gharib, 2008). Hence, there is a pressing need to correctly identify the nature of such nodules. FNAB, which is currently considered as the gold standard for evaluating thyroid nodules, significantly helps avoid unnecessary thyroidectomies for patients with benign thyroid lesions and improves the diagnostic accuracy of malignancy in the majority of cases (70\%) (Hamberger et al., 1982; Hadi et al., 1997; Castro and Gharib, 2003; Nayar and Ivanovic, 2009). However, it has limitations for determining malignancy in indeterminate nodules (up to $30 \%$ of FNAB results), leading to unnecessary surgery for the majority of indeterminate nodules (Nayar and Ivanovic, 2009). In order to improve the diagnostic accuracy of FNAB cytology in indeterminate lesions, miRNA assays have been investigated in recent years and have proven to be a promising second-line diagnostic tool in distinguishing malignant from benign thyroid nodules (Keutgen et al., 2012; Kitano et al., 2011, 2012; Shen et al., 2012; Vriens et al., 2012; Mazeh et al., 2011, 2013). However, different accuracies and conflicting results were obtained in different research studies. For instance, Keutgen et al. (2012) suggested that the results using a panel of 4 miRNAs (miR-328, miR-21, miR-197, and miR-222), which yielded a sensitivity of $86 \%$ and a specificity of $85 \%$ in discriminating malignant from benign nodules, were higher than those obtained from a panel of 2 miRNAs (miR-21 and miR-328), which yielded a sensitivity of $71 \%$ and a specificity of $85 \%$, or than those obtained using the individual miR-21, with a sensitivity of $69 \%$ and a specificity of $86 \%$, indicating that panels consisting of multiple miRNAs possessed higher diagnostic accuracy. In addition, Shen et al. (2012) found that the diagnostic utility of miR-221, miR-146b, miR-187, and miR-30d in FPTC, PTC, FTC, and ATC could differentiate malignant from benign lesions with a sensitivity of $93.2 \%$ and a specificity of $93.8 \%$. However, when the 4 miRNAs were used in FPTC, PTC, and FTC without ATC, the sensitivity dropped to $88.9 \%$ and the specificity dropped to $78.3 \%$, suggesting that the relative frequencies of FTCs might have an influence on the results of diagnostic value estimation, and also that it may be more difficult to distinguish FTCs from benign lesions. Thus, we conducted this meta-analysis to evaluate the diagnostic accuracy of miRNAs in differentiating malignant from benign nodules based on FNAB samples.

In our study, the diagnostic accuracy of single miRNAs was lower than that of multiple miRNAs. Two possible explanations may account for this result. First, the initiation and development of a malignancy consists of complex, multi-step molecular events, making a multiple miRNA assay a more complete indicator of these processes, and severely limiting the sensitivity and specificity of single miRNAs. As shown in our study, when multiple miRNA assays were utilized, the sensitivity increased from 0.75 to 0.90 , the specificity increased from 0.71 to 0.86 , the PLR increased from 2.71 to 6.14 , and the NLR decreased from 0.36 to 0.13 . Furthermore, the DOR had an improvement from 8.81 to 44.5 and the AUC made progress from moderate accuracy $(0.82)$ to relatively high accuracy $(0.95)$, indicating the high diagnostic value of a multiple miRNA panel as a potential screening tool to enhance thyroid cancer detection. Second, since the effect of benign disease processes on miRNA expression is not fully understood, use of a control group consisting of patients with benign thyroid disease may have an impact on the relative performance of single and multiple miRNA assays.

There are several limitations to our study. First, as we had emphasized that FNAB failed to identify indeterminate lesions, we made efforts toward improving the accuracy of differentiating malignant from benign lesions within the indeterminate group. However, one of the records included used only indeterminate FNAB samples for analysis (Keutgen et al., 2012), and others such as Kitano et al. (2012) used indeterminate FNA samples, among oth- 
er samples, as controls. Therefore, more research conducted with only indeterminate FNAB samples as controls is needed. Second, in the study of Shen et al. (2012), we noticed that when the relative frequencies of FTCs in the training sample set was lower than those in the validation sample set, the accuracy increased from 85.3 to $93.3 \%$. For PTC cases, Shen et al. (2012) yielded a diagnostic accuracy of $95.8 \%$, and Mazeh et al. (2011) also obtained similar results. Meanwhile, Keutgen et al. (2012) reported 4 miRNAs (miR-328, miR-21, miR-197, and miR222 ) in their study that yielded a specificity of $86 \%$ in differentiating malignant indeterminate lesions from benign ones based on FNAB samples, which could be improved to $95 \%$ if Hürthle cell neoplasms were excluded. Thus, different histological subtypes contained in the studies without discrimination may have led to different accuracies as the ability of miRNAs to identify them were different; therefore, further refinement in subtypes is needed. Third, the studies included did not mention whether the FNAB samples were the same samples evaluated by the cytologist, except for Mazeh et al. (2013), leading to the possibility that sampling error from separate biopsies might have impacted the accuracy of the miRNA assays. Finally, significant between-study heterogeneity was reflected by the $R$ index, so the random-effect model was chosen in our study; however, meta-regression was not conducted to explore the potential sources of heterogeneity due to the limitations of the studies included.

In summary, we identified that the use of multiple miRNAs could separate benign from malignant lesions in FNAB samples. Furthermore, a multiple miRNA assay with high specificity and sensitivity could potentially serve as clinically useful non-invasive biomarkers in thyroid cancer. However, further studies are warranted to confirm our findings and to explore the diagnostic potential of miRNAs in classifying indeterminate lesions of thyroid cancer.

\section{REFERENCES}

Adeniran AJ, Theoharis C, Hui P, Prasad ML, et al. (2011). Reflex BRAF testing in thyroid fine-needle aspiration biopsy with equivocal and positive interpretation: a prospective study. Thyroid 21: 717-723.

Banks ND, Kowalski J, Tsai HL, Somervell H, et al. (2008). A diagnostic predictor model for indeterminate or suspicious thyroid FNA samples. Thyroid 18: 933-941.

Castro MR and Gharib H (2003). Thyroid fine-needle aspiration biopsy: progress, practice, and pitfalls. Endocr. Pract. 9: $128-136$.

Chudova D, Wilde JI, Wang ET, Wang H, et al. (2010). Molecular classification of thyroid nodules using highdimensionality genomic data. J. Clin. Endocrinol. Metab. 95: 5296-5304.

Dean DS and Gharib H (2008). Epidemiology of thyroid nodules. Best Pract. Res. Clin. Endocrinol. Metab. 22: 901-911.

Engels BM and Hutvagner G (2006). Principles and effects of microRNA-mediated post-transcriptional gene regulation. Oncogene 25: 6163-6169.

Faquin WC and Baloch ZW (2010). Fine-needle aspiration of follicular patterned lesions of the thyroid: Diagnosis, management, and follow-up according to National Cancer Institute (NCI) recommendations. Diagn. Cytopathol. 38: 731-739.

Gharib H, Papini E, Valcavi R, Baskin HJ, et al. (2006). American Association of Clinical Endocrinologists and Associazione Medici Endocrinologi medical guidelines for clinical practice for the diagnosis and management of thyroid nodules. Endocr. Pract. 12: 63-102.

Hadi M, Gharib H, Goellner JR and Heerden JA (1997). Has fine-needle aspiration biopsy changed thyroid practice? Endocr. Pract. 3: 9-13.

Hamberger B, Gharib H, Melton LJ 3rd, Goellner JR, et al. (1982). Fine-needle aspiration biopsy of thyroid nodules. Impact on thyroid practice and cost of care. Am. J. Med. 73: 381-384.

Higgins JP, Thompson SG, Deeks JJ and Altman DG (2003). Measuring inconsistency in meta-analyses. BMJ 327: 557-560. Kato MA and Fahey TJ 3rd (2009). Molecular markers in thyroid cancer diagnostics. Surg. Clin. North Am. 89: 1139-1155.

Keutgen XM, Filicori F, Crowley MJ, Wang Y, et al. (2012). A panel of four miRNAs accurately differentiates malignant from benign indeterminate thyroid lesions on fine needle aspiration. Clin. Cancer Res. 18: 2032-2038. 
Khan SY, Tariq MA, Perrott JP, Brumbaugh CD, et al. (2013). Distinctive microRNA expression signatures in protonirradiated mice. Mol. Cell Biochem. 382: 225-235.

Kitano M, Rahbari R, Patterson EE, Xiong Y, et al. (2011). Expression profiling of difficult-to-diagnose thyroid histologic subtypes shows distinct expression profiles and identify candidate diagnostic microRNAs. Ann. Surg. Oncol. 18: 3443-3452.

Kitano M, Rahbari R, Patterson EE, Steinberg SM, et al. (2012). Evaluation of candidate diagnostic microRNAs in thyroid fine-needle aspiration biopsy samples. Thyroid 22: 285-291.

Kouniavsky G and Zeiger MA (2010). Thyroid tumorigenesis and molecular markers in thyroid cancer. Curr. Opin. Oncol. 22: $23-29$.

Layfield LJ, Cibas ES and Baloch Z (2010). Thyroid fine needle aspiration cytology: a review of the National Cancer Institute state of the science symposium. Cytopathology 21: 75-85.

Lee JC, Zhao JT, Clifton-Bligh RJ, Gill A, et al. (2013). MicroRNA-222 and microRNA-146b are tissue and circulating biomarkers of recurrent papillary thyroid cancer. Cancer 119: 4358-4365.

Lewis CM, Chang KP, Pitman M, Faquin WC, et al. (2009). Thyroid fine-needle aspiration biopsy: variability in reporting. Thyroid 19: 717-723.

Martinez I and Dimaio D (2011). B-Myb, cancer, senescence, and microRNAs. Cancer Res. 71: 5370-5373.

Mazeh H, Mizrahi I, Halle D, Ilyayev N, et al. (2011). Development of a microRNA-based molecular assay for the detection of papillary thyroid carcinoma in aspiration biopsy samples. Thyroid 21: 111-118.

Mazeh H, Levy Y, Mizrahi I, Appelbaum L, et al. (2013). Differentiating benign from malignant thyroid nodules using micro ribonucleic acid amplification in residual cells obtained by fine needle aspiration biopsy. J. Surg. Res. 180: 216-221.

Nayar R and Ivanovic M (2009). The indeterminate thyroid fine-needle aspiration: experience from an academic center using terminology similar to that proposed in the 2007 National Cancer Institute Thyroid Fine Needle Aspiration State of the Science Conference. Cancer 117: 195-202.

Pallante P, Visone R, Croce CM and Fusco A (2010). Deregulation of microRNA expression in follicular-cell-derived human thyroid carcinomas. Endocr. Relat. Cancer 17: F91-104.

Reddy SD, Gajula RP, Pakala SB and Kumar R (2010). MicroRNAs and cancer therapy: the next wave or here to stay? Cancer Biol. Ther. 9: 479-482.

Shen R, Liyanarachchi S, Li W, Wakely PE Jr, et al. (2012). MicroRNA signature in thyroid fine needle aspiration cytology applied to "atypia of undetermined significance" cases. Thyroid 22: 9-16.

Shibru D, Chung KW and Kebebew E (2008). Recent developments in the clinical application of thyroid cancer biomarkers. Curr. Opin. Oncol. 20: 13-18.

Siegel R, Ma J, Zou Z and Jemal A (2014). Cancer statistics, 2014. CA Cancer J. Clin. 64: 9-29.

Stang MT and Carty SE (2009). Recent developments in predicting thyroid malignancy. Curr. Opin. Oncol. 21: 11-17.

Subramanyam D and Blelloch R (2011). From microRNAs to targets: pathway discovery in cell fate transitions. Curr. Opin. Genet. Dev. 21: 498-503.

Visone R, Pallante P, Vecchione A, Cirombella R, et al. (2007). Specific microRNAs are downregulated in human thyroid anaplastic carcinomas. Oncogene 26: 7590-7595.

Vriens MR, Schreinemakers JM, Suh I, Guerrero MA, et al. (2009). Diagnostic markers and prognostic factors in thyroid cancer. Future Oncol. 5: 1283-1293.

Vriens MR, Weng J, Suh I, Huynh N, et al. (2012). MicroRNA expression profiling is a potential diagnostic tool for thyroid cancer. Cancer 118: 3426-3432.

Walter SD (2002). Properties of the summary receiver operating characteristic (SROC) curve for diagnostic test data. Stat. Med. 21: 1237-1256.

Wang CC, Friedman L, Kennedy GC, Wang H, et al. (2011). A large multicenter correlation study of thyroid nodule cytopathology and histopathology. Thyroid 21: 243-251.

Whiting P, Rutjes AW, Reitsma JB, Bossuyt PM, et al. (2003). The development of QUADAS: a tool for the quality assessment of studies of diagnostic accuracy included in systematic reviews. BMC Med. Res. Methodol. 3: 25.

Wiemer EA (2007). The role of microRNAs in cancer: no small matter. Eur. J. Cancer 43: 1529-1544.

Wilmott JS, Zhang XD, Hersey P and Scolyer RA (2011). The emerging important role of microRNAs in the pathogenesis, diagnosis and treatment of human cancers. Pathology 43: 657-671.

Zamora J, Abraira V, Muriel A, Khan K, et al. (2006). Meta-DiSc: a software for meta-analysis of test accuracy data. $B M C$ Med. Res. Methodol. 6: 31. 